\title{
In-Situ High Pressure Studies on Nanocrystalline Mercury Sulphide
}

\author{
K. M. Freny Joy ${ }^{1}$ and N. Victor Jaya ${ }^{2}$ \\ 1. Department of Physics, Jeppiaar Engineering College, Chennai 600 119, India \\ 2. Department of Physics, Anna University, Chennai 600 025, India
}

\begin{abstract}
Nanocrystalline $\beta-\mathrm{HgS}$ has many technological applications and prepared by novel microwave assisted method. The structural stability was studied by in situ high pressure X-ray powder diffraction measurements. No structural transformation was observed in nano-HgS upto $15 \mathrm{GPa}$. There is a shift in transition pressure towards the higher side when compared with the bulk materials. The volume decreased with an increase of pressure.
\end{abstract}

Keywords: Microwave heating, mercury sulphide, phase transition, high pressure XRD.

\section{Introduction}

Nanocrystalline materials with dimension less than $100 \mathrm{~nm}$ show remarkable revolution in the area of research in Physics, Chemistry and Engineering [1]. The metallic nanoparticles and their sulphide derivatives have become important components in various fields like catalysis [2], environmental remediation [3], gene therapy [4], imaging [5], drug delivery [6], biomarkers [7], sensors [8] and energy storage [9]. The unique properties of nanoparticles like large surface area, high magnetism and high chemical activity [10] paved way for the above said applications. These properties can be altered by the size and shape of the nanoparticles [11]. Sulphide semiconductors have been the focus of many researchers in recent years due to the quantum size effect [12, 13]. Among the semiconductor nanoparticles, Mercuric Sulphide (HgS) is a technologically important semiconductor and is widely used for application in ultrasonic transducer [14, 15], electrostatic image material [14], photoelectric conversion devices [16-18], acousto-optical materials [19] and infrared sensing

Corresponding author: K. M. Freny Joy, Dr., research fields: high pressure physics, nano synthesis and characterization.
[20]. Meta-cinnabar $(\beta-\mathrm{HgS})$ is an insulator which can be used for low power consumption electronic devices [21]. Research has been employed for the remediation of mercury and $\beta-\mathrm{HgS}$ is a promising nanoparticle to remove the toxicity of the mercury from the contaminated environment.

For this purpose $\beta$-HgS must be synthesized in various forms with high purity of controlled size and shape for stabilizing surface modification. Though we have a number of methods to prepare metal sulphide $(\mathrm{HgS})$ it is still a challenge to synthesize nanosized $\beta-\mathrm{HgS}$ particle by a practical and facile route. The microwave assisted route is a novel method which is faster and energy efficient. This method is used for synthesizing metal sulphide [22, 23]. It is a fast, convenient, mild, energy-efficient and environmentally friendly route for synthesizing $\beta-\mathrm{HgS}$. The prepared product was characterized by XRD, TEM and XPS. According to TEM observations the $\mathrm{HgS}$ nanoparticles are mostly spherical in shape with an average size of 15-20 $\mathrm{nm}$ [24].

High pressure plays a vital role to explore the phase transformation and possible path to expand the range of available solid state materials for applications. $\beta$-HgS being an II-VI semiconductor, the electrical transport property and structural property of $\mathrm{HgS}$ 
under high pressure is very important and useful. In our present work, a high pressure investigation is carried out in order to understand the structure of $\mathrm{HgS}$ under high pressure. The transition pressure obtained by experiment for the nanoparticle is compared with the transition pressure given in the literature for bulk materials

\section{Experimental Procedure}

\subsection{Sample Preparation}

About $1.5 \mathrm{~g}$ of mercury acetate $\left(\mathrm{Hg}\left(\mathrm{Ac}_{20}\right)\right)$ is added to $60 \mathrm{~mL}$ of polyethylene glycol (PEG-200). All the reagents used in the experiment are of analytical grade and used without any purification. About $0.2 \mathrm{~g}$ of sulphur powder is introduced into the PEG solvent. The amount of entire reagent is optimized. The mixture is then placed in the microwave refluxed system and the reaction is performed under ambient air for 20 minutes. The microwave oven followed a working cycle of 9 seconds "on" and 21 seconds "off" i.e. $30 \%$ power. At the end of the reaction, the black precipitates are collected and centrifuged, washed with distilled water and absolute acetone and finally dried in air. The final products are collected for characterization using XRD, TEM and XPS. According to TEM observation thus prepared, $\mathrm{HgS}$ nanoparticle is mostly spherical in shape. The average size of $\mathrm{HgS}$ is in the range $15-20 \mathrm{~nm}$. They are in good agreement with those estimated by the Debye-Scherrer equation. The XPS is employed to investigate the composition and purity of the prepared nanoparticles. The Hg:S ratio is $58: 42$ which shows that the surface of the products is rich in sulphur [24].

\subsection{High Pressure XRD}

The high pressure studies were carried out using a Mao-Bell type diamond anvil cell. The Rigaku $12 \mathrm{~kW}$ rotating anode X-ray generator with an energy dispersive X-ray diffraction system was employed. The samples were pressurized between the faces of two gem-quality diamonds made with brilliant cut.
The face diameter of the diamond anvil is about 600 $\mu \mathrm{m}$. The samples were placed in between the diamonds with the help of a stainless steel plate of grade T301 which sets as gasket. A hole of $0.3 \mathrm{~mm}$ was drilled at the centre of the indentation mark produced by the two diamonds. The sample was placed in the hole along with a 4:1 methanol-ethanol pressure transmitting medium. The sample was pressurized to various pressures up to $15 \mathrm{GPa}$. The pressure was measured in situ by placing silver powder along with the sample. The EOS of silver is used to determine the pressure.

\section{Result and Discussion}

At normal conditions the XRD patterns collected show that the products obtained are in cubic phase. The peaks obtained match very well with the literature patterns of $\beta-\mathrm{HgS}$ [25]. At the ambient pressure the peaks are indexed to the cubic phase in accordance with literature. On increase of pressure, we found that the peaks are slightly shifted along the lower value of energy. The energy and intensity of some peaks decreased on increasing the pressure. About $10 \mathrm{GPa}$, some peaks like (311) and (331) tend to diminish from vicinity. This confirms that as pressure is increased, the nanocrystalline phase of $\mathrm{HgS}$ transforms to the amorphous phase.

Generally $\mathrm{HgS}$ is observed under normal conditions both in the cinnabar phase (" $\alpha$ " or "red" form) and the metastable zincblende phase ("black" or " $\beta$ " form). At high pressure it has been reported that the phase transformation from zinc blende to cinnabar phase takes place at 5.5 GPa to $14.5 \mathrm{GPa}$ [26] and at $27 \mathrm{GPa}$ it transforms into rock salt $(\mathrm{NaCl})$ [27]. There is also evidence for a continuous transition to a phase III which appears to be a distorted form of $\mathrm{NaCl}$ at $<55$ $\mathrm{GPa}$ [27] and above $30 \mathrm{GPa}$ [28]. Based on the literature, the cubic phase transforms to distort $\mathrm{NaCl}$ at about $55 \mathrm{GPa}$. The above results have been reported for bulk HgS. No structural transition is obtained for nano sized $\mathrm{HgS}$ up to $15 \mathrm{GPa}$. Instead, we are able to 


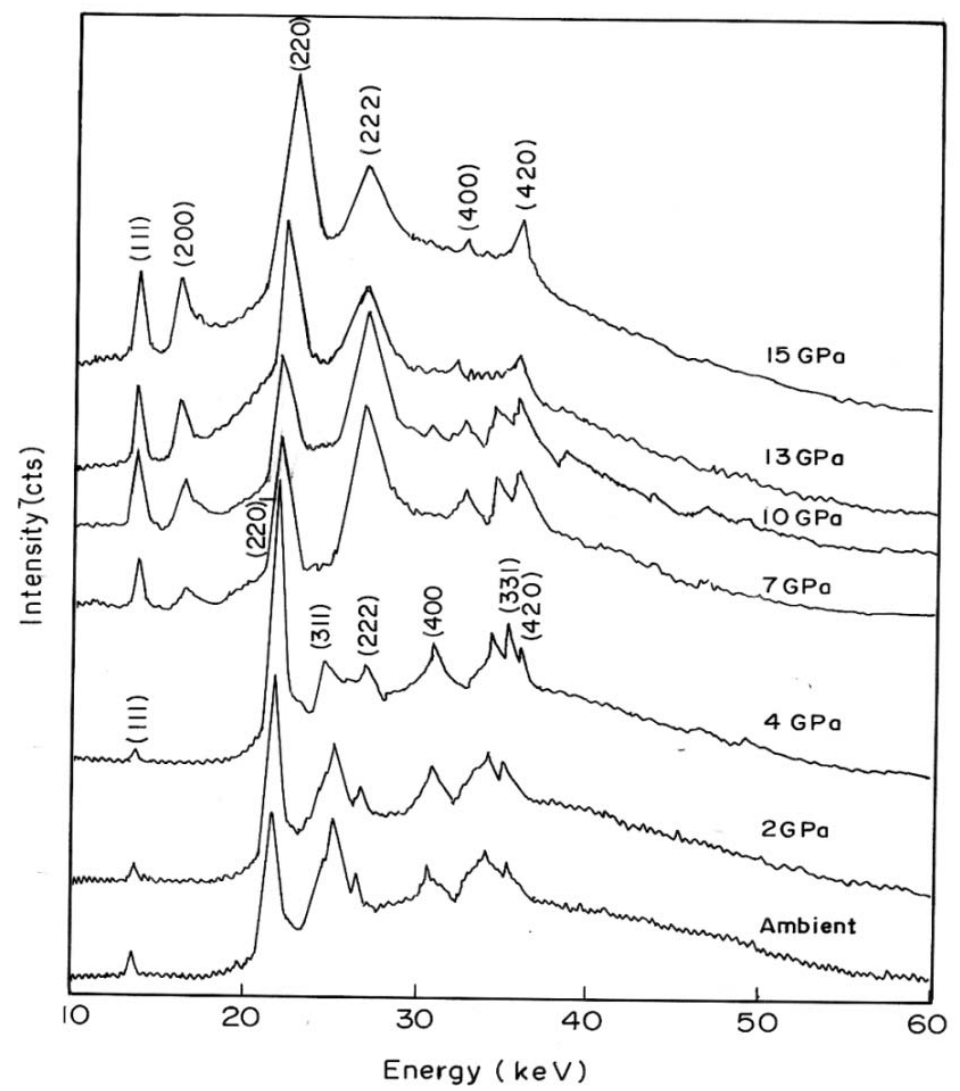

Fig. 1 EDXRD pattern of nano $\beta$-HgS for different pressures.

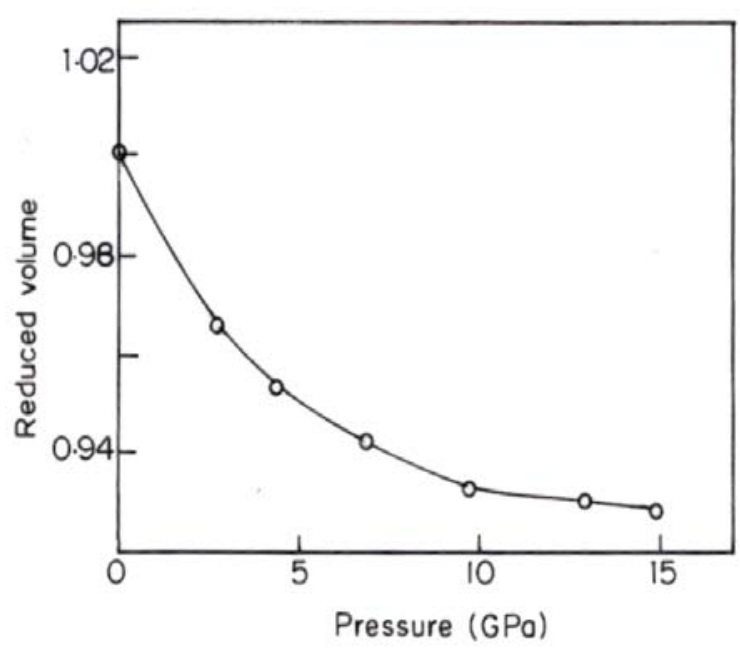

Fig. 2 Pressure-volume graph of nano $\beta$-HgS.

notice the disappearance of some peaks. Fig. 1 shows the high pressure XRD pattern of $\mathrm{HgS}$ with a particle size $20 \mathrm{~nm}$. The lattice parameter $\mathrm{a}=5.8621 \AA$ and volume is found to be $201.499 \AA^{3}$ at ambient pressures. On increase of pressure, the volume decreases following the universal accepted statement. Fig. 2 shows the pressure versus volume graph of nano $\mathrm{HgS}$ which is drawn in accordance with the Brich equation of state. For bulk $\mathrm{HgS}$, one must obtain a first-order phase transition in between 13-20 GPa. In the case of nanocrystalline $\mathrm{HgS}$, the first order is not seen up to $15 \mathrm{GPa}$.

The surface to volume ratio and the probability of mono domain crystallites formation is inversely proportional to the size of the nanocrystallite. The difference in the transition pressure between the bulk and the nanoparticle is comprised by three main factors [29, 30] such as (1) difference of surface energy of the phase under investigation, (2) difference of the internal energy at the crystallite site, (3) difference in the volume of the bulk and the nanoparticle. The surface energy plays a major role in the enhancement of transition pressure and phase transformation dynamics. The driving force for the 
phase transformation is the thermo dynamical reduction of Gibbs free energy from the initial phase to the final phase. Single nucleation effect also plays a vital role in the enhancement of transition pressure. This effect can be explained with the help of surface energy and shape changes. The peak width before and after the transition is the same on the application of pressure. This indicates that the nanocrystals transfer coherently without domain facture. In a solid-solid phase transformation, there is often a specific path that the atom follows when going from one phase to next phase [31, 32]. This path acts as the reaction coordinate for the phase transition. If all the atoms are moving in a well-defined path, this must cause the crystal to change shape. This is not usually observable in macroscopic crystals since the internal energy for bulk materials is contributed by each and every crystallite separately and cause multiple nucleation which in turn break the crystal into many domains, thus obscuring any overall change in shape. The shape change will change the position of atoms on the surface as well as in the interior and thereby alter the surface energy of the crystal. In bulk, most of the atoms are in the interior and change in surface energy is negligible. However, in nanocrystalline materials the internal energy is contributed form the core atoms as well as from the surface which on cumulative effect produce a mono nucleation. Moreover in nanocrystals a large fraction of the atoms is on the surface and this results in shape change. From this it is evident that volume collapse (nucleation) and surface energy difference contribute to the elevation of transition pressure in semiconductor nanocrystals.

\section{Conclusion}

The nanocrystalline mercury sulphide prepared by microwave assisted method is subjected to high pressure and no structural phase transformations are observed up to $15 \mathrm{GPa}$. The reduction of particle size has enhanced the transition pressure which in turn increases the surface energy. Due to the application of pressure the volume is reduced and the compressibility has enhanced. The volume collapse, internal energy and surface to volume ratio play a vital role in enhancing the transition pressure.

\section{Reference}

[1] Gleiter, H. 2000. "Nanostructured Materials, Basic Concept and Microstructure." Acta Materialia 48(1): 6243-8.

[2] Ansari, F., Grigiriev, P., and Labor, S. 2009. "DBT Degradation Enhancement by Decorating Rhodococcus Erythropolis IGST8 with Magnetic $\mathrm{Fe}_{3} \mathrm{O}_{4}$ Nanoparticles." Biotech Bioeng 102: 1505-12.

[3] Lukhele, L. P, Bhekie, B. M., Momba, M. N. B., and Krause, R. W. M. 2010. "Water Disinfection Using Novel Cyclodextrin Polyurethanes Containing Silver Nanoparticles Supported on Carbon Nanotubes.” J. Appl. Sci. 10: 65-70.

[4] Andreu, A., Fair, W. N., and Miller, A. D. 2008. "Clostridium Neurotoxin Fragments as Potential Targeting Moieties for Liposomal Gene Delivery to the CNS." Chem Bio Chem 9: 219-31.

[5] Lee, J. H., Huh, Y. M., and Jun, Y. W. 2006. “Artificially Engineered Magnetic Nanoparticles for Ultra-Sensitive Imaging." Nature Med 13: 95-9.

[6] Akin, D., Sturgis, J., and Ragheb, K. 2007. "Bacteria-mediated delivery of nanoparticles and Cargo into Cells." Nature Nanotech 2: 441-9.

[7] Ranzomi, A., Sabatte, G., Van Ijzendoorn, I. J., and Prins, M. W. 2012. "One Step Homogenous Magnetic Nanoparticle Immunoassay for Biomarker Detection Directly in Blood Plasma." ACS Nano 6: 3134-41.

[8] Fan, Y., Xu, S., and Schaller, R. 2010. "Nanopaticle Decorated Anodes for Enhanced Current Generation in Microbial Electrochemical Cells." Biosens Bioelectron 26: 1908-12.

[9] Ryu, J., Kim, S. W., Kang, K., and Park, C. B. 2010. "Synthesis of Diphenylalanine/Cobalt Oxide Hybrid Nanowires and Their Application to Energy Storage." ACS Nano 4: 159-64.

[10] (a) Akbarzadeh, A., Samiei, M., and Davaran, S. 2012. "Magnetic Nanoparticles: Preparation, Physical Properties and Application in Biomedicine." Nanoscale Res. Lett. 7: 144-56; (b) Chirita, M., and Grozescu, L. 2009. " $\mathrm{Fe}_{2} \mathrm{O}_{3}$-Nanoparticles, Physical Properties and Their Photochemical and Photoelectro Chemical Applications." Chem. Bull. 54: 1-8.

[11] Tao, A. R., Habas, S., and Yang, P. 2008. "Shape Control of Colloidal Metal Nanocrystals." Small 4: 310-25.

[12] Gorer, S., and Hodes, G. 1994. "Quantum Size Effects in 
the Study of Chemical Solution Deposition Mechanism of Semiconductor Films.” J. Phys. Chem 98 (20): 5338-46.

[13] Empedocles, S. A. 1999. "Influence of Spectral Diffusion on the Line Shapes of Single CdSe Nanocrystallite Quantum Dots." J. Phy. Chem 103 (11): 1826-30.

[14] Tokyo, N. 1975. Jpn Kokai Pat. 75130378 (Cl. H011L. C01b).

[15] Tokyo, N., and Aziko, K. 1978. Jpn. Kokai Pat.7855478 (C1. C23C15/00).

[16] Charkraborty, I., Mitra, D., and Moulik, S. P. 2005. "Spectroscopic Studies on Nanodispersions of CdS, $\mathrm{HgS}$ Their Core-Shells and Composites Prepared in Micellar Medium.” J. Nanopart. Res. 7: 227-36.

[17] Kershaw, S. V., Harrison, M., Rogach, A. L., and Kornowski, A. 2000. "Development of IR-Emitting Colloidal II-VI Quantum Dots Materials.” IEEE J. Sel. Top. Antum Electron 6: 534-43.

[18] Roberts, G. G., Lind, E. L., and Davis, E. A. 1969. "Photoelectronic Properties of Synthetic Mercury Sulphide Crystals." J. Phys. Chem Solids 30: 833-44.

[19] Sapriel, J. 1971. "Cinnabar ( $\alpha-\mathrm{HgS})$, a Promising Acousto-Optical Material.” Appl. Phys. Lett 19: 533-5.

[20] Higginson, K. A., Kuno, M., Bonevich, J., Qadri, S. B., Yousuf, M., and Mattoussi, H. 2002. "Synthesis and Characterization of Colloidal $\beta-\mathrm{HgS}$ Quantum Dots." J. Phys. Chem B 106: 9982-5.

[21] Virot, F., Hayn, R., Richter, M., and Vanden Brink, J. 2011. "Metacinnabar ( $\beta-\mathrm{HgS})$ : A Strong 3D Topological Insulator with Highly Anisotropic Surface States." $J$. Phys. Review Letter 106: 236806-1-4.

[22] Wang, H., Zhang, J. R., and Zhu, J. J. 2001. “A Microwave Assisted Heating Method for the rapid Synthesis of Sphalrite-Type Mercury Sulfide Nanocrystals with Different Sizes.” J. Cryst. Growth 233: 829-31.
[23] Ding, T., and Zhu, J. 2003. "Microwave Heating Synthesis of $\mathrm{HgS}$ and PbS Nanocrystals in Ethanol Solvent." Mater. Sci. Eng. 100: 307-13.

[24] Ding, T., Zhang, J. R., Long, S., and Zhu, J. J. 2003. "Synthesis of $\mathrm{HgS}$ and $\mathrm{PbS}$ Nanocrystals in a Polyol Sovent by Microwave Heating." Microelectron Engg 66: 46.

[25] JCPDS (Joint Committee on Powder Diffraction Standards) File No. 6-0261.

[26] Hao, A., Gao, C., Li, M., Chunyuan, H., Huan, X., Zhang, D., Yu, C., Liu, H., Ma, Y., and Tian, Y. 2007. “A Study of the Electrical Properties of $\mathrm{HgS}$ under High Pressure." J. of Phys: Cond. Matt. 19: 425222.

[27] Nelmes, R. J., and McMahon, M. I. 1998. "Structural Transitions in the Group IV, III-V, II-VI Semiconductors under Pressure." Semicond Semimetals 54: 145-246.

[28] Huan, T. L., and Ruoff, A. L. P. 1985. "High Pressure Induced Phase Transitions of Mercury Chalcogenides." Phy. Rev. B. 31: 5976-83.

[29] Tolbert, S. H., Herhold, A. B., Brus, E., and Alivisatos, A. P. 1996. "Pressure-Induced Structural Transformations in Si Nanocrystals: Surface and Shape Effects." Phys. Rev. Letts 76: 4384-7.

[30] Tolbert, S. H., and Alivisatos. 1995. "The Wurtzite to Rock Salt Structural Transformation in CdSe Nanocrystals under High Pressure." J. Chem. Phys 102: 4642.

[31] Chandi, D. J., and Martin, R. M. 1976. "Calculation of Lattice Dynamical Properties from Electronic Energies: Application to C, Si, and Ge." Sol. St. Commn. 19: 643-6.

[32] Weinstein, B. A. 1979. "High Pressure Phonon Dispersion of the Zinc Chalcogenides and the Metallic Transition." In High Pressure Science and Technology, edited by Timmerhaus, K. D., and Barber, M. S. New York: Plenum, 141-51. 